\title{
Proactive learning for intelligent maintenance in Industry 4.0
}

\author{
Rami Noureddine $^{1}$, Wei Deng Solvang ${ }^{1}$, Espen Johannessen ${ }^{1}$ and Hao $\mathrm{Yu}^{1}$,
}

\begin{abstract}
Manufacturing companies require efficient maintenance practices in order to improve business performance, ensure equipment availability and reduce process downtime. With the advent of new technology, manufacturing processes are evolving from the traditional ways into digitalized manufacturing. This transformation enables systems and machines to be connected in complex networks as a collaborative community through the industrial internet of things (IIoT) and cyber-physical system (CPS). Hence, advanced maintenance strategies should be developed in order to ensure the successful implementation of Industry 4.0, which aims to transform traditional product-oriented systems into product-service systems (PSS). Today, machines and systems are expected to gain selfawareness and self-predictiveness in order to provide management with more insight on the status of the factory. In this regards, real-time monitoring along with the application of advanced machine learning algorithms based on historical data will enable systems to understand the current operating conditions, predict the remaining useful life and detect anomalies in the process. This paper discusses the necessity of predictive maintenance to achieve a sustainable and service-oriented manufacturing system and provides a methodology to be followed for implementing proactive maintenance in the context of Industry 4.0.
\end{abstract}

Keywords: Anomaly detection, Predictive maintenance, Industry 4.0, Adaptive learning, Data Analytics

\section{Introduction}

Maintenance plans and policies are one of the most important decisions for all production and manufacturing processes. Companies have been implementing different maintenance activities and strategies in order to improve their overall performance in terms of production costs, wastes, flexibility, time and reliability.

The traditional ways of maintenance have evolved over time with the introduction of new technologies. The earliest maintenance activities are known as reactive maintenance activities where the management or workers deal with problems only when they occur. With the development to a higher maturity level, companies switch to preventive

\footnotetext{
${ }^{1}$ Rami Noureddine, Wei Deng Solvang, Espen Johannessen and Hao Yu ( $\square$ ) Department of Industrial Engineering, Faculty of Engineering Science and Technology, UiT The Arctic University of Norway, Narvik, Norway

\{rno034, wei.d.solvang, espen.johannessen, hao.yu\}@uit.no
} 
maintenance where frequent visualization by team members are scheduled and routine inspections of the system are essential to prevent the failure of equipment.

With the introduction of electronics and the widespread use of sensors and processors, many companies are able to adopt a higher level of maturity in maintenance by using rule-based predictive maintenance strategies. In rule-based maintenance, sensors are installed in some areas to measure specific parameters. A condition or a rule is coded to the sensor so that the system can send an alert to notify the management of the present status if the monitored parameters reach a predefined point.

However, with today's new technologies and the concept of a smart factory for Industry 4.0, maintenance is expected to reach much higher dimensions in terms of both maturity and efficiency. The main concept behind maintenance in Industry 4.0 is the ability to make use of historical and live data and the ability to make predictions of future states of processes. Besides, data visualization, digital twins, and augmented reality are also new technologies and concepts that provide companies with highly advanced efficient systems in maintenance and other activities in a production process.

The rest of the paper is organized as follows. Section 2 defines the concept and enabling technologies of industry 4.0. Section 3 discusses the benefits and the structure needed for predictive maintenance. Section 4 presents an approach for adaptive learning and predictive maintenance. Section 5 concludes the paper.

\section{Industry 4.0: Enabling technologies}

Industry 4.0 refers to the fourth industrial revolution with the introduction of big data, internet of things (IoT) and cyber-physical system (CPS). Today, many companies are competing to apply industry 4.0 methodologies in order to enhance their business performance. Companies and manufacturers are investing significantly in building up global networks to connect their machinery, factories and facilities in order to enable efficient communication and application of CPS.

The industrial internet of things (IIoT) in industry 4.0 has become attractive to many businesses due to the reduction in costs of computations, storage and network systems by the use of the cloud-computing model. The recent developments of an IoT framework and the advancements in the sensor technologies have established an integrated network that tightly connects systems and humans together [1]. IIoT systems can be effectively used to create operational smart factories in which a higher level of efficiency can be reached. IIoT objects, i.e., sensors, can be widely used to gather data in real-time from the field for improving the productivity through advanced automatic processes [2], improving the safety through a deeper knowledge of workers' position [3] and reducing the equipment failure through fast event detection capabilities [4].

Civerchia et al. [5] describes one architecture for an IIOT system, as shown in Figure 1. Sensors are used to measure the desired data from the environment. The Gateway is a node in the network, which is able to interact with the monitoring sensors and the mind of the system. Information is then passed wirelessly to the RCSR in the main 
control room to store data, which can be used for data analysis with advanced algorithms, pattern recognition, and data visualization. Finally, the OPC server stores all the data for authorized client access.

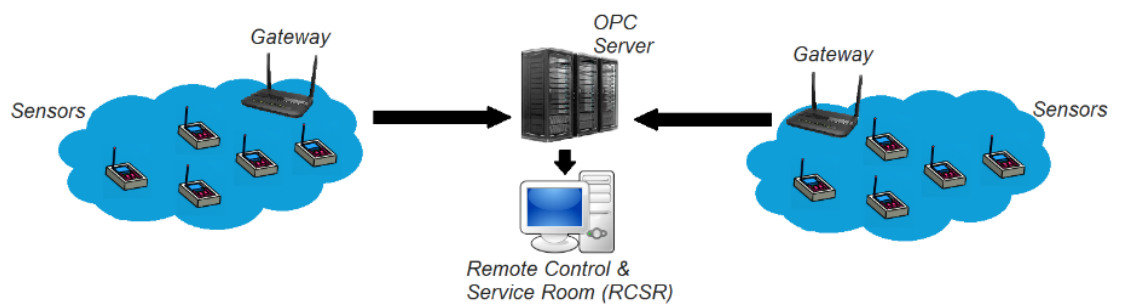

Figure 1: IIOT NGS-PlantOne architecture [5].

In IIOT systems, big data can be analyzed online via a cloud with advanced analytics at a very high speed, which can then be used by process engineers to obtain valuable information. As a result, future industry will be able to reach a high intelligence level by being capable of sharing this useful information across different nodes in the network and reacting to different conditions and events in an optimal way through CPS.

The concept behind CPS in Industry 4.0 is that they are intelligent systems containing embedded circuits that are connected to their environment. They do not only respond to the predefined stimulus, but also are able to communicate and interact with the surrounding environment. CPSs are networked and are thus able to send and receive data from different locations. CPS allows the construction of applications that can autonomously interact with environment and execute actions accordingly. Figure 2 shows the CPS framework for self-maintenance machines.

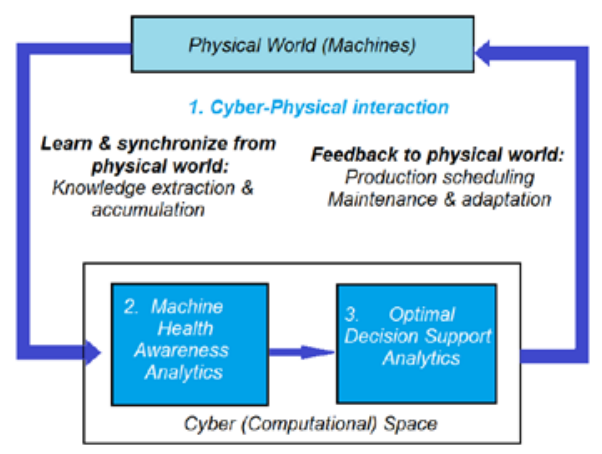

Figure 2: CPS framework for self-aware and self-maintenance machines [1].

Finally, it is important to know that the cloud in Industry 4.0 provides everything as a service. Three main categories are given as follows.

- Infrastructure as a service (IaaS): in which the hardware needed and the server rooms are presented as a service rather than buying it.

- Platform as a service (PaaS): gives access to the development languages, libraries, APIs, etc. 
- $\quad$ Software as a service (SaaS): provides services by providing users with web server-based shared application instead of accessing a copy of the application hosted in a local private server.

\section{Predictive maintenance supportive structure}

Predictive maintenance in Industry 4.0 is a method that enables just-in-time (JIT) maintenance. It can be used to prevent failure in process or machines by analyzing the operational data and identifying the patterns in order to predict issues before they occur. Using appropriate sensor installations, various signals, i.e., vibration, pressure, etc., can be extracted. In addition, historical data can be harvested for further data mining [1]. Value fetched from efficient data mining can then be utilized to drastically reduce the cost of maintenance and to improve the product quality, productivity, and profitability of production plants [6].

Information gathered in real-time can not only be applied to detect anomalies and predict future behavior by discovering the trends and the patters but also be used for improving the maintenance through the enhancement of design, installation, scheduling, and work procedures [7].

Some benefits obtained with the efficient use of predictive maintenance include:

1) Reduced maintenance time: maintenance is only performed when necessary.

2) Increased efficiency: unnecessary maintenance is reduced and root cause analysis becomes easier and automatic.

3) Improved customer satisfaction: alerts are sent to customers to inform the product status and to give suggestions regarding the product health.

4) Competitiveness: companies will gain a competitive advantage in the market by differentiating the products and brand.

In order to transform a traditional production plant into a smart factory, companies have to prepare an appropriate structure that can achieve and sustain the goal of it. As a result, several basic components and tools should be invested in order to make the manufacturing systems become more intelligent.

These components and tools include:

- Sensors: should be installed in the system in order to monitor the behavior and encode the system performance, efficiency, and status.

- Data-analysis tools: are needed to enable a root cause analysis

- Analytic algorithms: should be used to enable the predictive maintenance and smart diagnostics

- A communication system: is needed for the safe data storage and data transfer among different machines and teams.

- $\quad$ A central place for data storage: is needed, which can be either indoors or cloud based.

The structure allows data to flow from the production process to the central data storage area where the data from different systems and devices are gathered. Afterwards, the data is sent into machine learning algorithms for extracting knowledge, features, patterns, classes, and relations. After the data has been processed by machine 
learning algorithms, the results are sent to dashboards for visualizing the system status and predicting the future behavior. In addition, the messages or alarms are sent to respective people at the right time in order to notify an even that has happened or is about to happen in the production process. Data also flows in the reverse direction where the output of the machine learning algorithms can be used as the inputs for autonomous decision-makings. Figure 3 shows the predictive maintenance structure in Industry 4.0. It is an updated structure of the existing models, which only consider the one-way forward flow of information between different levels within a company.

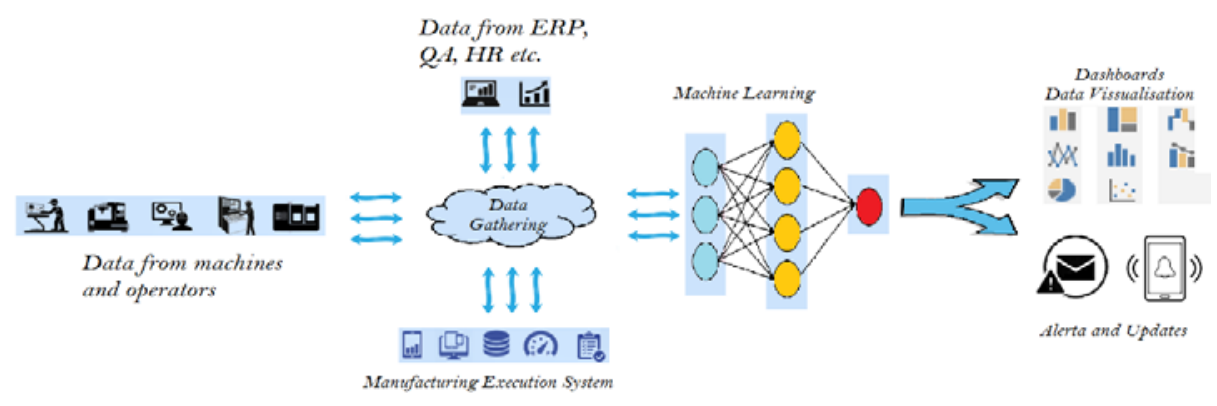

Figure 3: Industry 4.0 predictive analytics structure.

Machine learning algorithms used for predictive maintenance in Industry 4.0 vary in their characteristics and functions. There is not a specific algorithm type, which is applicable to all conditions. Each situation requires a specific algorithm depending on the characteristics, i.e., known or unknown functional form of the system, labeled or unlabeled data, deterministic or stochastic data, etc. For instance, a good algorithm for detecting the sequence behavior or time related behavior may consider the use of recurrent neural networks or even a combination of several algorithms, as done by Yuan et al. [8], in order to predict anomalies in rotating bearings behavior of a hydropower plant.

\section{$4 \quad$ Adaptive learning approach and applications}

The ability of continuous learning in real-time monitoring allows for reliable application of intelligent maintenance systems in manufacturing companies. This requires CPS to use adaptive learning techniques to continuously update the knowledge base for data mining.

When considering predictive maintenance and health assessment of machines or systems in real life application, the systems need to be able to adapt to new emerging conditions that are prone to happen rather than the traditional way of feeding the information to the algorithms prior to operations. As shown in Figure 4, an approach to enable such adaptive learning is discussed by Lee et al. [1].

The proposed approach considers the use of unsupervised machine learning algorithms, i.e., SOM or GMM, to read the input data in a certain state of the system and measure the difference comparing to the existing states in the knowledge base. If the 
state is measured to be close to an existing state or cluster in the system, the knowledge base is updated with the new data. While if no similar cluster is found, a new cluster is added to the knowledge base in order to account for the new behavior.

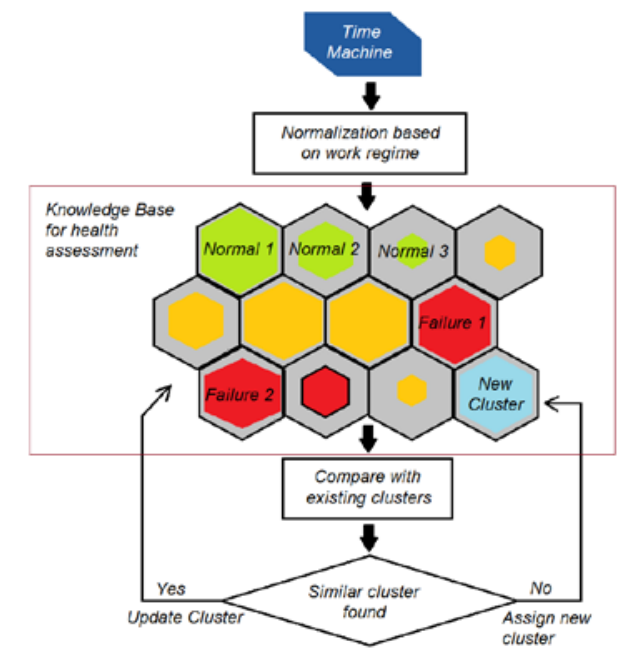

Figure 4: Adaptive learning methodology [1].

With the increase on the complexity of machines and systems, the root cause analysis for undesirable events or failures becomes a very difficult task. Several factors, i.e., personnel related tasks and operating conditions of equipment, etc., may all be the influencing factors of certain outcomes. In this regards, one highlighted benefit of applying the predictive maintenance in industry 4.0 is the ability to discover the underlying patterns in the gathered data and to model the systems using the data-driven approach in order to provide solutions for an effective root cause analysis and an automated response.

Our solution suggests the manufacturing process can be continuously improved by using an adaptive learning predictive maintenance approach with constant updates of the knowledge base of the system based on detected undesirable or abnormal states, maintenance personnel information, and troubleshooting data.

The first step is to gather the relevant data that is expected to have an effect on the system. Expert opinions are needed in order to decide which data should be included and how they are measured. The second step requires data cleansing and outlier removal for consistent analysis. In the third step, missing data is estimated by different techniques, i.e., moving average. In the fourth step, detected faults are gathered and added to the historical data. Based on which, machine learning algorithms i.e., the Naïve Bayesian filter, neural networks, etc., can be used to detect how the outcome is affected by the change of data. These algorithms can provide helpful solutions due to their capabilities to model complex nonlinear processes with a high level of confidence. Finally, the algorithm performance should be validated before they can be applied in the real operations. 
Since the reaction of different personnel to different problems is also gathered and stored for analysis. The CPS is able to assign different problems to the right person by mobile or email notifications based on smart decisions. These decisions may consider the expertise, speed, distance as well as other factors for each individual.
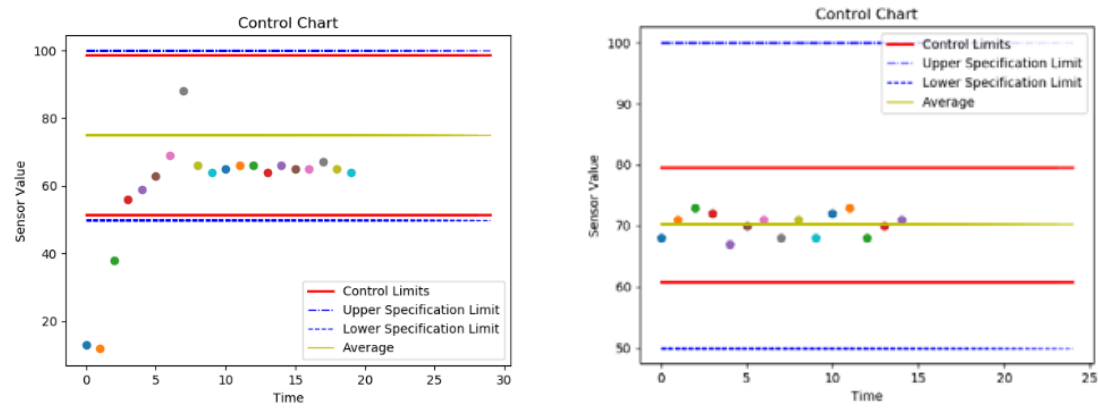

Figure 5: Updates of the control limits based on the real-time data (left) and the data measured by an ultrasonic sensor using a new approach (right).

The ability to include adaptive learning in manufacturing processes using the technologies in Industry 4.0 can also result in better designing and operational procedures, which will further lead to a continuous improvement of the process. Figure 5 shows how control limits have been updated in real-time and reflects how the process variation have been reduced by different practices. The system can then send alerts to the relevant person or take autonomous decisions in order to ensure the process is operated in an optimal way.

\section{Conclusion}

New technologies in Industry 4.0 are paving the way for new practices and techniques to be used in the maintenance operations. The proposed concept of Industry 4.0 maintenance is characterized by the ability to derive knowledge from historical data and by the ability to learn from new live data in real-time.

To successfully realize the goals of industry 4.0, equipment in a smart factory should possess self-awareness for current-condition evaluation and future-condition prediction. Thus, the idea of proactive and predictive maintenance becomes extremely important for reducing the maintenance time and unnecessary disrupting checkups, improving efficiency by increasing the asset life, enhancing working environment, and creating new revenue streams by supporting product-service systems [9].

One successful example of product-based servitization is the "Total Care" scheme established by Rolls-Royce [10], where a proactive maintenance strategy has enabled both parties to lower down the costs while increasing effectiveness. Technicians are able to monitor the key performance indicators in aircraft engines and use proactive maintenance techniques to support a continuous and sustainable value creation process. 
This research provides a framework for the implementation of predictive maintenance in Industry 4.0 and describes the structure needed to support predictive maintenance. Furthermore, the methodology of adaptive learning is introduced in order to enable the identification of the root causes and the optimization of alerting notifications. Last but not the least, the paper also shows how proactive learning can help manufacturers to optimize their performances by monitoring the process variations and updating control limits in order to improve the standardization in the system.

\section{Acknowledgement}

This research is supported by the OptiLog 4.0 project financed by the Research Council of Norway (Grand no. 283084).

\section{Reference}

1. Lee J, Kao HA, Yang S. Service innovation and smart analytics for industry 4.0 and big data environment. Procedia Cirp. 2014 Jan 1; 16:3-8.

2. Breivold HP, Sandström K. Internet of things for industrial automation--challenges and technical solutions. In 2015 IEEE International Conference on Data Science and Data Intensive Systems 2015 Dec 11 (pp. 532-539). IEEE.

3. Petracca M, Bocchino S, Azzarà A, Pelliccia R, Ghibaudi M, Pagano P. WSN and RFID Integration in the IoT scenario: An Advanced safety System for Industrial Plants.

4. Wang J, Zhang L, Duan L, Gao RX. A new paradigm of cloud-based predictive maintenance for intelligent manufacturing. Journal of Intelligent Manufacturing. 2017 Jun 1;28(5):112537.

5. Civerchia F, Bocchino S, Salvadori C, Rossi E, Maggiani L, Petracca M. Industrial Internet of Things monitoring solution for advanced predictive maintenance applications. Journal of Industrial Information Integration. 2017 Sep 1; 7:4-12.

6. Mobley RK. An introduction to predictive maintenance. Elsevier; 2002 Oct 24.

7. Dhillon BS. Engineering maintenance: A modern approach. cRc press; 2002 Feb 14.

8. Yuan J, Wang Y, Wang K. LSTM Based Prediction and Time-Temperature Varying Rate Fusion for Hydropower Plant Anomaly Detection: A Case Study. In International Workshop of Advanced Manufacturing and Automation 2018 Sep 20 (pp. 86-94). Springer, Singapore.

9. Ferreiro S, Konde E, Fernández S, Prado A. Industry 4.0: Predictive Intelligent Maintenance for Production Equipment. In European Conference of the Prognostics and Health Management Society, no 2016 Jun (pp. 1-8).

10. Yu H, Solvang WD. Enhancing the competitiveness of manufacturers through Small-scale Intelligent Manufacturing System (SIMS): A supply chain perspective. In2017 6th International Conference on Industrial Technology and Management (ICITM) 2017 Mar 7 (pp. 101107). IEEE. 